\title{
"II SENTIMENTO
}

\section{DELIA TRADIONEM}

\section{LUZI LEGGE}

UNGARETHI

Rosanna Pozzi*

pozzi.rosanna@virgilio.it

Università dell'Insubria

Fecha de recepción: agosto de 2016

Fecha de aceptación: diciembre de 2016

Resumen: Il saggio passa in rassegna le riflessioni critiche di Mario Luzi a proposito di Giuseppe Ungaretti, scritte e pubblicate tra il 1952 e il 2000, mettendone in luce le tre fondamentali chiavi d'interpretazione: la religiosità nel suo nesso con il dolore e la morte; l'esperienza di sradicamento dalla terra d'origine e la conseguente ricerca di recupero della tradizione letteraria italiana; l'origine della

* Rosanna Pozzi è Laureata in lettere classiche presso Università di Genova nel 1993 con una tesi sulla pièce teatrale Ipazia di Mario Luzi, attualmente è docente di letteratura italiana e latina presso il Liceo Scientifico Statale Arturo Tosi di Busto Arsizio (VA) e Cultrice della materia presso la Facoltà di Scienze della comunicazione dell'Università dell'Insubria- Dipartimento di Scienze teoriche e applicate; ha conseguito il titolo Dottore di ricerca presso l'Università di Genova (Dipartimento F.I.S.T.I.R.), con una tesi dedicata agli scritti critici di Mario Luzi. È stata Visiting Professor di Letteratura Universale presso UCSS Lima- Perù nel luglio 2007. Il suo ambito di studio prevalente riguarda il teatro in versi di Mario Luzi, i testi critici di Mario Luzi su poeti italiani del Novecento e il nesso arte poesia nelle liriche di Mario Luzi e nelle sue prose d'arte; si è occupata inoltre della collaborazione di Mario Luzi con il Corriere della Sera. In occasione del centenario della nascita del poeta ha raccolto in Nove poeti per Mario Luzi (Aracne, 2014) altrettante interviste ad alcuni tra i maggiori poeti italiani contemporanei. Nel centenario della Grande Guerra si è dedicata ad aspetti particolari della pubblicistica di guerra (Giornali di Trincea dopo Caporetto). 
sua poesia dall'esperienza concatenata di morte, vita, "giubilazione". L'autrice intreccia le riflessioni luziane con il contributo critico di altri estimatori della poesia ungarettiana, quali Mengaldo, Ramat, Cortellessa, Bigongiari, Papini, Zanzotto, Sereni.

Palabras clave: Mario Luzi, interpretazione critica, poesia, tradizione letteraria, religiosità, Ungaretti

\section{GTHE FEELING OF TRADITION" LUZI READS UNGARETTI}

Abstract: The present essay makes a review of the critical reflections of Mario Luzi about Giuseppe Ungaretti, written and published from 1952 to 2000, highlighting three key points of their interpretation: the religiosity, considered in its relationship with pain and death; the experience of being uprooted from his homeland and the consequent search for a recovery of the Italian literary tradition; the origin of his poetry in the concatenated experience of death, life, "jubilation". The author interweaves Luzi's reflections with the critical contributions of other admirers of Ungaretti’s poetry, as Mengaldo, Ramat, Cortellessa, Bigongiari, Papini, Zanzotto, Sereni.

Keywords: Mario Luzi, critical reflections, poems, Italian litterary tradition, religiosity, Ungaretti

$\mathrm{G}$ li scritti critici di Mario Luzi a proposito di Giuseppe Ungaretti sono certamente densi e originali per il punto di vista introdotto sul poeta, del quale mettono in luce i seguenti aspetti essenziali: la religiosità nel suo nesso con il dolore e la morte; l'esperienza di sradicamento e la conseguente ricerca di recupero della tradizione letteraria italiana; l'origine della sua poesia nell'esperienza concatenata di morte, vita, giubilazione.

Il primo scritto luziano su Ungaretti, datato 1952, fu significativamente pubblicato in un'Antologia della poesia religiosa italiana cotemporanea nella quale Luzi forniva anche una brevissima nota biografica, non riportata nelle successive sedi di pubblicazione, che si trascrive per completezza documentaria in nota ${ }^{11}$. Nell'articolo, utilizzato come introduzione alla selezione di liriche ungarettiane, l'accento era posto sulla tematica religiosa, in conformità

1 Nel Profilo di Ungaretti, contenuto nella Antologia della poesia religiosa italiana contemporanea, si legge: “Giuseppe Ungaretti è nato ad Alessandria d'Egitto nel 1888 da genitori toscani. Fu a Parigi; visse la guerra di trincea dal ' 15 sino alla fine. Ha pubblicato il primo libro di versi nel' 16 col titolo Il porto sepolto. È considerato uno dei massimi poeti della letteratura contemporanea. L'edizione definitiva delle sue opere va stampandosi e sono già uscite le raccolte di poesia L'allegria, Milano 1943; Sentimento del tempo, Milano 1943; Poesie disperse, Milano 1945; Il dolore, Milano 1947; La terra promessa, Milano 1949. Altri testi e traduzioni sono usciti recentemente. Vive a Roma dove insegna all'Università' (Luzi, 1952, p. 134). 
non solo con il tipo d'antologia nella quale veniva pubblicato ma anche e soprattutto con l'intenso e drammatico contenuto religioso espresso nella sua poesia. In seconda sede di pubblicazione, infatti, con un titolo fortemente connotato, Religiosità come evasione, Luzi rendeva esplicito in partenza l'anelito metafisico del poeta; se ne riporta l'esordio per chiarire e meglio comprendere la lirica ungarettiana secondo il punto di vista luziano:

Sento un certo imbarazzo nel parlare della religiosità di Ungaretti; e dipende dal fatto che essa non comporta una visione definita, né un ordine di valutazione coerente. Si potrebbe dire in sostanza che il poeta non possiede alcuna delle certezze che la religione suole accordare a chi veramente ne è toccato; e neppure possiede quella misura a cui il pensiero dell'uomo religioso è informato. La religiosità di Ungaretti compare soprattutto in certi culmini quando per un estremo di sofferenza egli sente il bisogno di rivolgersi in alto; il dolore strappa un grido e il poeta presume ci sia chi possa ascoltarlo. In questo senso rappresenta un'evasione dal finito; Ungaretti pone Iddio dove altri poeti videro l'infinito. (Luzi, 2002, p. 123)

La particolarità del rapporto con l'infinito di Ungaretti deriva, sempre secondo Luzi, "dal suo modo di sentire il finito che non è un modo contemplativo, ma una partecipazione violenta e infine insostenibile che esige poi il ricorso al Padre, al Salvatore" (Luzi, 2002, p. 123). È una religiosità dagli accenti drammatici e implacati, un anelito religioso correlato e derivato da un'esperienza di vita e di dolore intensi:

Ecco perché gli accenti religiosi sono così drammatici e, potremmo dire, implacati come la sua esperienza. In fondo allo sperimento dei sentimenti intensamente esercitati, in fondo al vano rovello della ragione, quando né quelli né questa possono più delimitare il senso della sofferenza provata, nasce in Ungaretti il ricorso a Dio — preghiera o apostrofe — si fa sentire il bisogno di provare la causa o la protezione. (Luzi, 2002, p. 123)

Pubblicato tre anni dopo l'uscita de Il dolore (1947) e ad un anno di distanza da la La terra promessa (1950), la breve riflessione luziana poneva in rilievo lo stretto legame 
tra religiosità e dolore, registrando il passaggio da un sentimento religioso vago ad uno più preciso e ben delineato nei suoi tratti di valenza cristica, come a tutti è noto, dapprima nel frangente della guerra e poi nel dramma della perdita personale e privata:

Nel volume che appunto s'intitola al dolore, l'accento religioso è più forte e, mi si perdoni il bisticcio, più religioso. Dio non è più un dato, puro e astratto, situato oltre i termini dell'esperienza, un elemento dell'estrema inevitabile dialettica dell'esistere umano; ma è sceso dentro i termini della vita spirituale e affettiva del poeta, ha preso un volto più preciso e più familiare; $\mathrm{e}$ se voi richiamate alla mente qual è da ultimo in Ungaretti il sentimento della tradizione, potrete senza troppa circospezione tradurre i due aggettivi in uno solo e dire cattolico. (Luzi, 2002, p. 124)

Luzi proseguiva nella medesima pagina chiarendo che la connotazione cattolica della religiosità ungarettiana si svela in modo chiarissimo e inequivocabile a partire da Mio fume anche tu, lirica nella quale, sempre in dipendenza dall'esperienza del dolore, emergono i lineamenti della divinità:

Nella celebre poesia su Roma occupata, Mio fume anche $t u$, mentre gli attributi della divinità si precisano ("Santo, santo che soffri”) e ne sono evocati i misteri nel senso direttamente cristiano ("Per liberare dalla morte i morti / E sorreggere noi infelici vivi”) il poeta non chiede più soltanto la certezza e il soccorso, ma celebra la connivenza nel dolore; e in questo sembra veramente riconoscersi e scoprirsi devoto (“D”un pianto solo mio non piango più, / Ecco, Ti chiamo, Santo / Santo, Santo che soffri”). È questo certamente il punto più chiaro della religiosità di Ungaretti. Essa si chiarifica per vie tutte umane. (Luzi, 2002, p. 124)

Per Luzi, infatti, la religiosità di Ungaretti non nasce come "un orientamento distinto dello spirito" (Luzi, 2002, p. 123), quanto piuttosto come "un'attitudine innata" (Luzi, 2002, p. 123), che si manifesta "nel drammatico senso di perdizione e di colpa, nell'orrore e nell'incarnazione dei sensi, in quel certo suo feticismo" (Luzi, 2002, p. 124). 
Vale la pena ricordare che la brevissima riflessione di Luzi veniva pubblicata in seconda sede sulle pagine della Fiera Letteraria, con il titolo di "Religiosità come evasione" nella rubrica intitolata "Galleria degli Scrittori Italiani", in quell'occasione interamente dedicata ad Ungaretti e articolata in quattro pagine per dare spazio e rilievo non solo al poeta, ma anche al traduttore, al critico, allo scrittore in prosa e alle notizie biografiche; il contributo luziano apparve accanto alle firme di Giuseppe De Robertis, Alfredo Gargiuolo, Emilio Cecchi, Piero Bigongiari, Emilio Gadda, Giovanni Papini e altri ancora. De Robertis, dedicandosi all' Allegria (1931), scriveva: “Queste pagine 'lavorano' dentro di noi. In apparenza povere, si arricchiscono a ripensarle, e ci arricchiscono" (De Robertis, 1953, p. 3); Emilio Cecchi annotava a sua volta su Il dolore che "la straordinaria natura poetica d'Ungaretti non si prova soltanto da ciò che egli scrive, ma dal suo modo di vivere e comunicare" (Cecchi, 1953, p. 3), mentre Piero Bigongiari annotava a proposito de La terra promessa (1950): "Del drammatico ha dovuto esaurire ogni tentazione fino a scoprire la resa lirica, rimanendo drammatico il presupposto" (Bigongiari, 1953, p. 3), mentre Papini riproponeva il primo articolo dedicato al poeta del Porto sepolto (1916), per recensire "le più care e sollevate poesie che abbia dato la guerra italiana" (Papini, 1953, p. 4).

A distanza di oltre vent'anni, nel 1979, Luzi tornava ad occuparsi del poeta nomade con un articolo intitolato Classicità e giubilazione di Ungaretti e delineava un percorso a tutto tondo nella sua lirica alla luce dell'intera produzione poetica ormai completa a quella data. In prima istanza evidenziava la familiarità del poeta con la dimensione della morte nelle sue varie declinazioni e forme:

A nessun poeta fondamentale la dimensione della morte è estranea. In Ungaretti ha preso varie forme; la remissione del naufrago, la sublimazione dell'eros, il desiderio di annullamento, la lucentezza cristiana del ricongiungimento con Dio. Tutte le forme tra cui si dibatte in sostanza la accesa pulsazione della vita, meglio che una precisa filosofia. (Luzi, 2002, p. 125)

Per Ungaretti il nesso con la morte è all'insegna della concretezza, affermava Luzi, in conformità "con il senso e il valore da attribuire definitivamente al lungo corso della poesia ungarettiana, che è di aver testimoniato l'uomo" (Luzi, 2002, p. 125); Luzi distingueva 
infatti nella cultura moderna tra "testimonianza dell'uomo" e "testimonianza dell'idea che l'uomo ha di sé" (Luzi, 2002, p. 125), ossia la distinzione tra sperimentare e teorizzare:

Nella cultura moderna l'uomo ha testimoniato l'idea che ha di sé, della sua situazione e della sua storia: la sua testimonianza è stata ideologica, mitologica, ipotesi. Ungaretti ha testimoniato la creatura-uomo, l'uomo in quanto creatura nei suoi movimenti elementari di desiderio e di sconfitta, di illuminazione e di oscurità: qualcosa di più umile e radicale dell'immagine dell'uomo o della stessa coscienza dell'uomo elaborate dalla cultura moderna. (Luzi, 2002, p. 125)

Il poeta toscano precisava, inoltre, che nelle sue liriche, soprattutto nell'Allegria, non si descrive l'uomo nell'accezione universale di umanità, ma l'uomo Ungaretti con la sua specifica individualità, con le sue personali e precise esperienze biografiche che vanno a formare un destino:

Quest'uomo, specialmente in un primo irripetibile momento che è quello di L'allegria non ha la U maiuscola, ma i semplici connotati individuali di lui, Giuseppe Ungaretti, a cui lo sradicamento, il conseguente nomadismo, la stanchezza e la saturazione di civiltà logorate danno una naturale investitura a significare la condizione critica dell'europeo contemporaneo prima e durante il conflitto. In questo caso circostanze, che per altri furono fortuite, si compongono in un vero destino: l'infanzia ad Alessandria d'Egitto, il contatto con la disgregata civiltà araba, la rivelazione del deserto e dei suoi misteriosi movimenti, la conoscenza poi di Parigi ormai fatta con quel deposito d'immagini irreversibili nella mente. (Luzi, 2002, p. 126)

Per capire meglio la lirica del primo Ungaretti alla luce dei dati biografici che lo riguardavano, Luzi rimandava all'intervista ${ }^{2}$ che Jean Amrouche gli aveva rivolto nel giugno

2 L'intervista di Jean Amrouche a Giuseppe Ungaretti, andata in onda sul Programma Nazionale Radiofonico il 6 giugno 1955 per il ciclo de "I colloqui con Jean Amrouche", appartiene ad una delle dodici interviste rivolte da Amrouche a Ungaretti nel corso della trasmissione curata dal giornalista tunisino per la Radio Nazionale francese dal 1944 al 1956, alla quale era solito invitare intellettuali, poeti, pittori, musicisti e artisti. L'intervista è stata poi raccolta in Ungaretti e Amrouche (1972, pp. 137-143); citazioni comprese tra p. 137 e p. 142. 
del 1955, andata in onda sul Programma Nazionale Radiofonico su Rai 3 de "L'Approdo", dalla quale si riportano di seguito alcuni passaggi, a partire dall'intento dichiarato del colloquio, ossia "intervistare l'illustre (...) Giuseppe Ungaretti sulla sua opera e sui rapporti che essa ha avuto con la sua vita", chiarendone anche la motivazione:

Mi si permetta di dire che per i francesi della mia generazione la poesia di Ungaretti fu una delle rivelazioni più profonde. Poesia semplice, ingenua come un canto naturale e nello stesso tempo sapiente, che colpisce direttamente il cuore e la mente. Poesia umana, fatta per tutti gli uomini, da un uomo che da sé si è definito: "Ungaretti uomo di pena”. (Ungaretti, 1972, p. 137)

Nel colloquio con Armouche si chiariva il primissimo legame dell'ispirazione lirica del poeta di Alessandria d'Egitto con il tema della morte e della pena, quando Ungaretti, ricordando le prime prove poetiche nel periodo ginnasiale, faceva riferimento ad un diario, poi stracciato, sul quale annotava dei versi, "dei motivi molto malinconici", ispirati a delle "nenie di ciechi che si tenevano per mano" (Ungaretti, 1972, p. 139), cantate nel corso di "funerali mussulmani, arabi(...)" (Ungaretti, 1972, p. 139), che passavano sotto la finestra del suo collegio; prove di versi alle quali neppure Ungaretti diede molta importanza e che in realtà erano preludio al suo successivo interesse per le lettere, osteggiato dalla famiglia, per ragioni economiche, come affermava nel corso dell'intervista:

Nella famiglia, quando più tardi io non volli fare quello che la mia famiglia avrebbe avuto desiderio che facessi...allora naturalmente ci furono delle opposizioni perché io ho voluto mettermi sulla via delle lettere, non era una cosa che a mia madre potesse piacere, ma insomma...la poesia o la letteratura non hanno mai dato pane, non molto pane a nessuno. Non lo danno ancora oggi, che sono diventato vecchio, in modo...sì, in modo sufficiente sì... in modo abbondante no! (...) Agli onori e alla gloria io non ci ho mai pensato. Gli onori o la gloria, sa, sono cose $\mathrm{da}(. .$.$) chi faccia poesia sul serio non ha mai$ pensato né agli onori, né alla gloria. Ha pensato di farla sul serio; che è una cosa molto grave, e molto difficile. (Ungaretti, 1972, p. 140) 
Ungaretti considerava che anche le poesie uscite su Lacerba "non erano buone poesie, non erano ancora il momento in cui c'era in me netta, chiara, la vocazione della poesia. Erano ancora così, dei tentennamenti, erano degli approcci ma... non erano ancora la poesia" (Ungaretti, 1972, p. 141), mentre ricordava quali suoi maestri e autori importanti e formativi già dalla scuola Leopardi e soprattutto Mallarmé:

Fin dalla scuola... è curioso... fino dalla scuola per esempio, Leopardi, già... è stranissimo insomma, è stranissimo, fino dalla scuola Leopardi e fino dalla scuola Mallarmé. Pare incredibile, ma io ho conosciuto Mallarmé, ho incominciato a leggere Mallarmé quando andavo ancora a scuola; e questo è merito dei miei maestri, Coller, uno svizzero, che ci leggeva il "Mercure de France"... Il "Mercure" in quell'epoca lì era una grande rivista, era una grande rivista, dalla quale tutti traevano insegnamenti in un senso o nell'altro...eh già, ci leggeva il "Mercure". E così venne fuori questo nome di Mallarmé. E chissà perché io mi sentii attratto, non capivo un gran che, sì certo, non capivo un gran che, ma insomma c'era un segreto in quella musica, c'era in quelle parole, c'era un segreto e sentivo che c'era... che quel segreto era la poesia, era la poesia... ed era verso quel segreto che dovevo andare. (Ungaretti, 1972, p. 142)

La prima fase poetica vera e propria di Ungaretti, a suo giudizio così come per Luzi, fu quella delle prime raccolte - Il porto sepolto (1916), L'allegria di naufragi (1919), L'allegria (1931) - nelle quali si attuò un'autodefinizione, attraverso liriche come Girovago e I fumi, solo per citarne alcune, e si delineò anche una doppia polarità di termini chiave, quali il "binomio nomadismo-paese innocente" (Luzi, 2002, p. 126), che "diventerà poi un trinomio quando il paese innocente, collocato primamente nell'innocenza della parola, si condenserà nell'immagine sogno della terra promessa" (Luzi, 2002, p. 126), una tripartizione all'insegna del viaggio, di un intinerario dapprima casuale poi destinato ad una meta. L“immagine-sogno” (Luzi, 2002, p. 126) della terra promessa, secondo Luzi, "è operante assai prima che si profili come tema esplicito e dia il nome alla tarda attività di Ungaretti" (Luzi, 2002, p. 126), ed è presente in modo "oscillante e multiforme" (Luzi, 
2002, p. 127), mentre "il nomadismo, per lo meno come traslato spirituale, continua identico” (Luzi, 2002, p. 127).

Infatti, nella già citata intervista di Jean Amrouche, alla domanda relativa ad una eventuale "rivolta dell'adolescenza" (Amrouche, 1972, p. 142) o piuttosto "rivolta metafisica" (Amrouche, 1972, p. 142), Ungaretti rispondeva invece introducendo il tema di uno "squilibrio" (Ungaretti, 1972, p. 142), derivato da una mancanza, originata dall'essere un italiano e un europeo "da lontano" (Ungaretti, 1972, p. 142):

Ah! Una rivolta metafisica... beh...io veramente di rivolte metafisiche... io ho capito, vedo a che cosa lei mira... naturalmente io sono nato lontano dall'Italia. Io ho avuto un'educazione italiana ma da lontano e anche non soltanto italiana, ma europea, ma da lontano, quindi io... è uno strano sentimento, insomma, ma per questo sentimento non si può parlare di rivolta. Ma di... come se fosse avvenuto un taglio... un taglio tra quello che è nostro e... le condizioni nelle quali si è così, per fatalità, costretti a vivere. E questa è una cosa che, di fatti, ha prodotto nel mio essere, e non soltanto nel mio essere, ha prodotto qualche cosa che nel mio essere metteva uno squilibrio. (Ungaretti, 1972, p. 142)

Puntualizzava poi la natura di tale "squilibrio" (Ungaretti, 1972, p. 142) definendolo come "un senso di sradicamento" (Ungaretti, 1972, p. 142):

Eh già, naturalmente... È il senso di sentirmi lontano da ciò che è mio, un senso di sradicamento no?! Io ero...ecco già è questo, questo...un senso... forse in tutta l'Europa c'era in quel periodo... era un periodo curiosamente felice, quello, in Europa, e me ne accorsi, e poi... stando a Parigi a lungo, era un periodo stranamente felice, stranamente... un periodo di euforia... apparentemente, ma nell'animo della gente c'era qualche cosa che faceva sentire... faceva sentire non so che...quella felicità reale, era così... era... sì, c'era la ricchezza, c'era... ma non si sa perché quelle cose lì... questo era generale, era in tutta l'Europa (...). Eppure c'era palesemente... c'era un'apparente felicità ma in fondo la gente era felice, e non si sa perché, c'era uno squilibrio interno; un po' sì, partecipavo di questo squilibrio, e un po' questo squilibrio era 
aggravato dal fatto che mi sentivo legato a una cultura dalla quale poi ero in qualche modo distaccato, e sulla quale s'era aggiunto qualche cosa di diverso. (Ungaretti, 1972, p. 142)

Amrouche cercava di identificare "questa cosa diversa" (Ungaretti, 1972, p. 142) con "il paesaggio africano, il deserto... questo paese... come si può dire, di nulla: il tempo infinito e il deserto infinito e il mare infinito..." (Amrouche, 1972, p. 141), e allora Ungaretti specificava meglio:

Sì, ma si tratta di cose che bisognerebbe distinguere. C'è il senso dell'uomo che ha il sentimento d'essere tagliato fuori dalla cosa alla quale però vorrebbe ricongiungersi e forse non potrà mai ricongiungersi interamente. C'era come una tradizione alla quale si sentiva il bisogno di riattaccarsi e dalla quale io ero separato, che dava questo stato... questo stato di disagio dell'animo. E poi naturalmente c'è la grande impressione che resterà presente in tutta la mia poesia, che è l'Africa, l'Africa, l'Africa mussulmana... l'Africa mussulmana resterà costantemente presente nella mia poesia con la sua profonda malinconia, con l'infinito e l'aridità del deserto, con il sentimento...con il sentimento...un sentimento bruciante, bruciante, eh, sì, ci sono tutte queste cose... e sono sempre rimaste vive... e il sole... il sole... il sole... tutte queste cose sono rimaste... non potevano non rimanere vive nella mia poesia... non potevano... ma insomma questo avverrà poi, questa è un'altra cosa. (Ungaretti, 1972, p. 142)

Si avverte in tale intervista il delinearsi di quegli elementi che hanno permesso a Luzi di individuare il trinomio nomadismo, paese innocente, terra promessa, proprio per il fatto che, partito dall'Africa per cercare le proprie radici in Italia, Ungaretti non si sentirà mai parte integrante del paese d'origine, identificato come "paese lontano, straniero", gli rimarrà per tutta la vita la nostalgia dell'infanzia africana ed esprimerà tale inquietudine, nostalgia, ricerca con l'espressione del tema e dello stato d'animo di viaggio verso la terra promessa: 
...straniero... va bene, era... quali legami sentivo... l'ho detto, sentivo in qualche modo, di essere stato tagliato dall'Italia, tagliato... tagliato da me stesso... e tagliato da me stesso sentivo questo... questo taglio... questo paese che era lontano da me... che era lontano nello spazio e lontano anche perché il mondo, il mondo intorno a me, il mondo delle prime emozioni, era un mondo diverso, non era l'Italia. Quindi questo naturalmente, questo creava in me un senso...questo senso di desiderio...di desiderio e di colmare questa specie di fosso che c'era tra me e il mio paese... questo senso di... e nello stesso tempo questo....anche questo amore per questo paese nel quale le mie emozioni erano nate..., le mie prime emozioni, quelle che durano tutta la vita, erano nate, in un paese che non sarebbe stato mail il mio... Certo, questo è presente costantemente nella mia poesia. (Ungaretti, 1972, p. 142)

Senza dubbio il soggiorno parigino lo avvicinò all'Europa, al centro culturale più vivo in quel momento, gli permise di conoscere intellettuali, pittori, poeti europei e italiani ma non fu quella la spinta propulsiva al nascere della sua poesia, né al suo avvicinarsi alla propria tradizione europea, fu un fatto negativo, drammatico e luttuoso, come ricorda Ungaretti in conclusione d'intervista, il suicidio dell'amico egiziano Sceab:

E dunque... dunque... sì, certo, il contatto con l'Europa: eh, già, il contatto con l'Europa, fu un contatto difficile nei primi tempi, nei primi contatti... sì, passai per l'Italia, non vi rimasi molto, i primi contatti li ebbi a Parigi. Erano gli anni nei quali stavano uscendo tutte le grandi tendenze di riforma dell'arte, delle lettere, a Parigi...C'erano stati anche Mallarmé; poi, dopo, anche, per la pittura c'era stato Cézanne, ma insomma era un periodo, era un bel periodo; e certo quei contatti che ho avuto a Parigi, contatti avuti con Delaunay, contatti avuti con Braque, contatti avuti con Apollineare, con Max Jacob, con Salmon, con tanti poeti anche italiani di passaggio a Parigi: Soffici o Papini o Palazzeschi eccetera, quei contatti certo erano importanti, ma non era quello che io cercavo. Io mi rendevo conto di tutti i progressi che il linguaggio poetico, il linguaggio delle arti stava facendo...di tutti i progressi che... nelle lettere e nelle arti conseguiva... me ne rendevo conto, ma insomma, 
probabilmente, tutte quelle ricerche non mi sono servite a nulla. Mi sono servite a farmi sentire che appartenevo...che appartenevo... a un mondo, che potevo facilmente appartenere a un mondo, legarmi a un mondo che poteva diventare il mio mondo, ma insomma non erano quelle le ricerche che poi avrebbero determinato la nascita vera al momento vero della mia poesia. (Ungaretti, 1972, p. 142)

Come è noto il suicidio fu indotto nel giovane egiziano proprio in conseguenza dell'esperienza di sradicamento dall'origine, come motiva Ungaretti, che per similarità di situazione a quel punto fu mosso a sentire la necessità di riattaccarsi ad una tradizione:

Questa tragedia è stata resa possibile dal fatto che Scheab, di origine araba, non si sentiva più legato alla sua cultura, alla cultura tradizionale, alla cultura dei suoi, e sentiva per quanto la possedesse a fondo, sentiva di non poter aderire a quella... interamente a quella che si era scelta, che era la cultura francese. E questo ha determinato il suo suicidio... Ed è naturale che questo possa avermi fatto sentire la necessità di riattaccarmi, costi quel che costi, a una tradizione: alla mia. (Ungaretti, 1972, p. 142)

Se il primo imput alla poesia fu il tragico episodio appena narrato, quella familiarità con la morte di cui Luzi scriveva in apertura d'articolo nel già citato Classicità e giubilazione, se la tematica della ricerca del paese dell'infanzia, del viaggio nomade e della terra promessa rimarranno costanti, se le suggestioni del soggiorno parigino lo misero in contatto con un mondo, una cultura e una tradizione senza però risultare determinanti per lo scaturire della sua poesia, come lo fu invece il suicidio dell'amico egiziano e poi l'esperienza della guerra, con Sentimento del tempo (1933), invece, secondo Luzi, "Ungaretti ha cessato di essere quel tipo di poeta che si è detto, immediatamente e quasi fatalmente significativo" (Luzi, 1979, p. 8076), in altri termini è passato da una spontaneità immediata e fresca al dialogo con la cultura, diventando un poeta colto, fino ad arrivare "a riscoprire il principio del lirismo" (Luzi, 1979, p. 8076): 
La spontanea esemplarità del suo nudo diario ha compiuto il suo destino. Ungaretti è diventato un poeta colto, cioè la sua poesia si decide ora nel dialogo con una cultura. Questa cultura è la cultura degli anni Venti, del ritorno al classico, della ripresa simbolista - sia pure sotto segno contrario. Valéry emerge al posto delle avanguardie, Picasso riscopre Pompei, Stravinsky eccita la sua invenzione nell'attrito con la tradizione illustre. "La Ronda" procede nella sua opera di restauro. Ungaretti lavora in profondità a riscoprire il principio del lirismo o, come ben diceva, del canto italiano e della sua durata nel tempo: è un lavoro importante per la cultura italiana. (Luzi, 2002, pp. 126-127)

In tale lavorio di approfondimento e ricerca "Ungaretti si complica o meglio libera in modi fortemente divaricati le sue componenti” (Luzi, 2002, p. 127):

Sentimento del tempo ha due anime: una sensuale, magica, erotica; l'altra interrogativa, problematica, cristiana: e l'intensità agglutinante, che ora si è sostituita all'integrità della parola, la esprime come due, in contrasto. La terra promessa prende lineamenti cangianti e perfino contraddittori. Le stazioni del viaggio si moltiplicano: l'isola smemorata, la magia erotica dello sperdimento, e poi di soprassalto il balenare di un'altra opposta qualità di "sonno felice" nella primavera eterna, nell'eterna umanità della redenzione che esige tutto un altro cammino. Permane l'idea di oasi, di riposo inalterabile, legata alle nozioni fondamentali e primarie di deserto, di nomadismo; ma la direzione, in termini del viaggio sono divergenti. (Luzi, 2002, p. 128)

Seguendo il discorso luziano inizialmente la terra promessa oscilla come un miraggio nelle liriche Dove la luce, La madre, Caino, poi ad un certo punto nell'officina creativa di Ungaretti "la terra promessa diventa un tema distinto di concentrazione" (Luzi, 2002, p. 128) l'idea ispiratrice di un poema, che prenderà in prima istanza la forma incompiuta $e$ mitologica del volumetto intitolato appunto Terra promessa (1950), rivisitazione parziale del mito di Enea e del suo viaggio, svolto solo "nel controcanto doloroso di Didone" (Luzi, 2002, p. 128), e in un secondo momento ne Il Taccuino del vecchio (1961), dove "non c'è più tensione verso il miraggio, ma sorda riflessione sulla natura di questo desiderio, 
disincantate conclusioni su questo affanno dell'uomo, provate, ancora una volta, sul vivo della propria individuale vicenda" (Luzi, 2002, p. 128).

Sembra che la lettura svolta da Luzi faccia concludere l'itinerario poetico e umano di Ungaretti secondo la traiettoria di una parabola discendente, sembra che "il nomadismo abbia tradito Ungaretti" (Luzi, 2002, p. 128), che "il viaggio si sia concluso nell'oscurità" (Luzi, 2002, p.128), poiché nel quinto coro del Taccuino si legge: "Si percorre il deserto con residui / Di qualche immagine di prima in mente, / Della Terra promessa / nient'altro un vivo sa" (Luzi, 2002, p. 128); e invece, in conclusione d'articolo, Luzi riafferma, motivandola, "la leggendaria allegria" (Luzi, 2002, p. 128) del poeta in oggetto, consistente "nell'umiltà e nell'innocenza con cui l'uomo subisce il suo scacco; soffre la sua condizione" (Luzi, 2002, p. 128):

Non avrebbe, lui, accettato questo sconforto. L'oscurità per Ungaretti non degradava l'uomo, né il senso catastrofico della colpa umana toglieva alla vita, il suo prodigio. Tutto sta nell'umiltà e nella innocenza con cui l'uomo subisce il suo scacco; soffre la sua condizione. La coscienza di aver vissuto senza difesa e protezioni di orgoglio, come creatura contraddittoria ma libera e di aver servito perfino nelle sue illusioni rientrate senza avvilirlo, dava a Ungaretti la sua leggendaria allegria. (Luzi, 2002, p. 128)

Luzi tornava sugli stessi temi, ossia sullo spaesamento e la tradizione in occasione del convegno di studi dedicato a Ungaretti nel 1981 a Urbino, con un intervento erroneamente intitolato La presenza l'attualità di Ungaretti e poi corretto nella riproposizione del già noto titolo Ungaretti e la tradizione, a ribadire con più convinzione e consapevolezza la centralità di alcune tematiche nella sua lettura e interpretazione del poeta, con l'intento d'approfondire e d'ampliare i sopracitati nuclei tematici, qui riproposti e ricapitolati, cui aggiunse una precisazione e una premessa. La precisazione riguardava l'attualità della poesia d'Ungaretti, ribadendo l'importanza del nesso morte / vita nella mossa primigenia della sua ispirazione poetica, così come la centralità del recupero della tradizione e la sua memorabilità nella produzione poetica; vi si legge infatti: 
Per i poeti si perpetua quel connubio e quel contrasto di molta morte e di molta vita: che è la loro condizione e direi, il loro mestiere; perché scrivere non si può se non al cospetto della morte, di ciò che è distrutto e che noi uccidiamo per una nuova vita. Attualità. Quale attualità? È un fatto poco conoscibile, poco commensurabile l'attualità di una poesia. Non dico l'attualità cosmica, ma proprio quella del retaggio e della trasmissione. Non si misura solo con il numero degli studi e delle esercitazioni dedicatele, dalla frequenza delle citazioni. Come nasce dalla profondità, agisce nella profondità: e questa azione emerge alla superficie talora quando è più allentata e, appunto, superficiale. Chi può dire chi riceve che cosa in questo momento? Tutte le forze sono in gioco e anche la poesia è una forza, una energia liberata... (Luzi, 1984, p. 42)

La premessa, invece, "è un dato semplice semplice, quasi ovvio, e cioè che in essa [nella poesia di Ungaretti] tutto è significato, tutto è valutato: non ci sono sospensioni della conoscibilità e del giudizio e meno ancora dell'espressività accettate come tali, voglio dire come connotato del mondo e della sua rappresentazione" (Luzi, 1984, p. 43). Luzi spiega meglio che la parola ungarettiana si dipana tra "volontà di legiferazione" (Luzi, 1984, p. 43), e "d'interrogazione" (Luzi, 1984, p. 43), in una tensione spasmodica a definire e ad esprimere nella parola il molteplice e il transeunte della realtà:

Più la parola è difficile, più si esalta della sua stessa difficoltà, più la caccia a fissare e a definire e a stringere il sostanziale e il transeunte della morsa di ciò che è detto è accanita. La volontà di legiferazione è almeno altrettanto forte che quella d'interrogazione: e Ungaretti legifera con il suo enunciato, non si trovano in lui altre forme di autorità o di subordinazione del genere che l'arte dubitativa ci ha nel frattempo abituato a conoscere. Non si fida se non di ciò che è catturato dalla frase, dal verso, detto, fosse anche iperdetto, scritto, fosse anche iperscritto con una volontà insieme di dettato e di scrittura che talvolta eccede sulla cosa e la soverchia. (Luzi, 1984, p. 44)

La parola di Ungaretti, secondo Luzi, tende ad indicare la realtà nella sua precisione assoluta, anche nelle più sottili sfumature, costituite da vocaboli e silenzi: 
L'accenno a un inseguimento non gli basta, si tratti pure di una nuance essa deve essere raggiunta ed eguagliata dalla finitezza della parola. Anche i famosi silenzi del testo ungarettiano sono in cornice: l'allusività del linguaggio analogico di cui tanto si è parlato non esclude il puntiglio della letteralità a oltranza, della letteralità-monumento. Ungaretti non mostra di fare affidamento su alcuna complicità, non aspetta collaborazioni dall'esterno o per meglio dire dall'interno di una cultura e di una sensibilità da cui, come ad altri è accaduto, si senta portato e sostenuto. (Luzi, 1984, p. 45)

Se Luzi sottolineava nell'intervento di Urbino il lavoro di scavo, di appropriazione del significato dei vocaboli insieme alla tradizione nella fase di stesura del testo poetico, soffermandosi sulla "solitudine del testo ungarettiano" (Luzi, 1984, p. 45) e sulla "solitudine della sua mente accentrata sulla nuda, ascetica operazione di assumere, di scrivere e di circoscrivere" (Luzi, 1984, p. 46), qualche tempo dopo in occasione della mostra documentaria e iconografica intitolata Ungaretti, la biblioteca di un nomade 3 , allestita dal Ministero dei Beni Culturali a Roma nel dicembre del 1997, veniva resa pubblica la ricca biblioteca del padre, grazie alla figlia, Anna Maria Ungaretti, con edizioni rarissime da Petrarca a Montale, ed emergeva in quella il diuturno lavoro di lettura, approfondimento, assimilazione dei testi della letteratura italiana chiosate a margine dallo stesso autore, spesso con l'annotazione d'intuizioni e spunti, poi ripresi altrove, così come altrettanto spesso con traduzioni in francese di corrispondenti vocaboli italiani, sconosciuti nel loro significato più ampio a un giovane italiano cresciuto in un collegio di impostazione e di cultura certo europea ma prevalentemente francofona: il francese, infatti, era la lingua veicolare per le lezioni in aula e per le comunicazioni in un ambiente internazionale ma di prevalente cultura arabo-francese.

\footnotetext{
L'intero catalogo è disponibile on-line in Internet Culturale. Cataloghi e collezioni digitali delle biblioteche italiane, versione digitale della mostra Ungaretti. La biblioteca di un nomade, allestita a Roma, Teatro dei Dioscuri; 10 dicembre 1997- 10 febbraio 1998. Il catalogo riporta le sezioni della mostra in questordine: Linfanzia; I contatti letterari; Ungaretti e l'Egitto; Ungaretti e Parigi; La prima guerra mondiale; Il porto sepolto; Dalla trincea a Parigi; Le quattro stagioni della poesia; Il sentimento del tempo; La conversione; Il Brasile; Il dolore; Gli ultimi lavori; Una biblioteca d'antenati. Fu organizzata con il patrocinio del Ministero per i Beni e le Attività culturali e la Direzione Generale per i Beni librari e gli Istituti Culturali.
} 
Per rendersi conto dell'importanza delle letture e dello scavo nella tradizione letteraria italiana da parte del poeta del Porto sepolto, si rimanda al catalogo della suddetta mostra alla sezione Una biblioteca di antenati, dove si legge:

Conservata con cura dagli eredi, la superstite Biblioteca di Ungaretti è testimone solo parziale delle letture del poeta. Molto infatti s'è smarrito, di trasloco in trasloco: il nomade, come amò definirsi, che fece della nascita africana il punto di forza, ha vissuto sotto cieli diversi e spesso con disagio. D'altra parte non si ignora né il suo apprendistato anche remoto, a cominciare dai libri maneggiati dall'adolescente in Egitto o da quelli che il giovane inconsapevole accumula durante il primo soggiorno parigino; né s'ignorano poi gli acquisti italiani e brasiliani dagli anni Venti agli anni Quaranta. Insomma, giù fino all'ultima vecchiaia, testimonianze dirette, saggistica e carteggi, insieme con le ricerche minute e mirate condotte da più parti, valgono a configurare in buona misura il patrimonio librario che è lo specchio della formazione e delle predilizioni di Ungaretti (...). È inoltre acquisito, grazie agli studi definitivamente probati di Carlo Ossola, che la Vita d'un uomo, sotto la cui insegna il poeta volle iscrivere la propria opera, è ben più cartacea di quanto la vistosa cifra vitalistica intenda suggerire. Si vuol dire cioè che il movente autobiografico della scrittura, esibita come diario, va certo ridimensionato a favore dell'essenza letteraria della parola ungarettiana. (Ministero dei Beni Culturali a Roma, 1997)

Si ribadisce l'importanza della tradizione, delle letture, del nesso letteratura-vita, spesso sottovalutato per l'apparente semplicità e libertà del primo Ungaretti, qualità attribuite o poste in linea di continuità con le Avanguardie o con uno spontaneismo vitalistico, in realtà dettate e derivate anche e piuttosto dall'urgenza dell'esperienza del dolore e della morte, ma ancor più da un poeta che non possiede una lingua e una tradizione certa, della quale va lentamente appropriandosi in controtendenza con i coevi poeti italiani che la stavano rifiutando, abolendo e distruggendo nei suoi modelli e nelle sue strutture formali, lessicali, logico-sintattiche; si prosegue la citazione precedente a riprova di quanto affermato:

I libri - dice Ungaretti - “spalancano la vita”. E proprio perché i suoi fiumi - Serchio e Nilo, Senna e Isonzo - sfociano in un mare d'inchiostro, il poeta 
è stato in grado di fondare da noi il linguaggio lirico di un secolo che inizia con le avanguardie: con gesti negativi, anche plateali, avversi alla tradizione. A colpi di manifesti ci si era scagliati contro l'Accademia e contro il Museo, mentre l'io era per la velocità e per la macchina, per la guerra... nel clima della rivoluzione futurista Ungaretti muove i primi passi, e per giunta nella Parigi di Apollineare; ma la patria di cui il nomade patisce la privazione agirà come un antidoto. Da sempre spaesato nel deserto natale dove la luce accesa è alla ricerca della terra densa di storia e fitta di ombre dei suoi avi. E il recupero difficile che la trincea rende urgente, lo conduce verso il passato che sembra travolto dagli eventi non meno che dagli anni. Non a caso la trafila romantica —Leopardi e Baudelaire in primo luogo- è il viatico dell'esordiente. Trova lì istanze eversive più forti delle marinettiane. Spetta a Ungaretti il primato come innovatore del linguaggio poetico appunto perché lo sorprendiamo curvo su altri poeti, sulla parola che l'ha preceduto, della quale rivendica l'eredità quasi in forma di preghiera. Così, mentre i poeti nostrani della sua generazione rifiutano, almeno nelle poesie, Pascoli e d'Annunzio, Ungaretti, al contrario, non manca di tributare a quei modelli l'omaggio devoto che negli anni non smentirà. (Ministero dei Beni Culturali a Roma, 1997)

I curatori della mostra ${ }^{44}$ ricordano inoltre che "Prezzolini, Papini o Soffici, quando sono all'altro capo del filo del soldato semplice ormai poeta battezzato, dovranno corrispondergli libri su libri. Non importa se 'usati', insiste Ungaretti, che tra i brandelli di muro, nell'aria crivellata come una trina, trova nelle letture la prima ragione di quell'attaccamento alla vita che è il messaggio di tutta la sua opera" (Ministero dei Beni Culturali a Roma, 1997) e annotano ancora in modo chiaro e certo che "la superstite Biblioteca attesta il ritorno di Ungaretti ai classici” (Ministero dei Beni Culturali a Roma, 1997).

Del resto, al rappel à l'ordre, degli anni Venti e oltre, si somma il ruolo di professore a cominciare dalla trasferta brasiliana. Apprestandosi ad attraversare

\footnotetext{
Il comitato scientifico era costituito da Rosetta Agresti Mosco, Annamaria Andreoli, Maria Grazia Benini, Maurizio Calvesi, Simonetta Carrozza, Luigi De Nardis, Paola Montefoschi, Mario Petrucciani, Leone Piccioni e Alexandra Zingone.
} 
l'Oceano per il nuovo incarico - siamo nel 1936 - il docente che non ha alle spalle un regolare corso di studi parte con un oneroso fardello di libri. Stilato diligentemente, l'elenco autografo di quella Biblioteca in miniatura (162 volumi) ha molto da dire sul progetto didattico. Veramente, se non sapessimo a chi appartiene quel corredo librario potremmo cadere in un errore di identificazione. Ecco un laureato — si direbbe- alla scuola di Monaci o di D'Ancona o di Ascoli... Se non che, la massiccia presenza della Scuola storica é temperata da quella di Croce. A colpo d'occhio, - negli anni contrassegnati dalla polemica sugli scartafacci, è il variantismo ad attrarre Ungaretti - dovunque esso si trovi. Si motiva così il fatto che uno dei corsi universitari brasiliani sia dedicato a Manzoni: tra Fermo e Lucia e Promessi Sposi può appagare un interesse preciso, tutto rivolto all'elaborazione e alla trasformazione del testo. (Ministero dei Beni Culturali a Roma, 1997)

Se nel 1997 nel catalogo delle mostra si legge di questo dialogo con la tradizione italiana e i suoi autori e si scopre con sorpresa che nella Biblioteca di Ungaretti Manzoni è uno degli autori prediletti e si apprende che la lettura dei Promessi sposi o degli Inni sacri fu attenta e puntuale, come attestano non solo le stesure manoscritte delle lezioni universitarie (ne restano ben sei, suddivise tematicamente), ma anche gli appunti fulminei del lettore che intende fissare subito, a caldo, ciò che in seguito svilupperà per esteso, già nel 1981 Luzi sosteneva che un campo d'indagine e di studio da considerare e approfondire fosse proprio quello del rapporto di Ungaretti con la tradizione, che è per lui "un termine fisso a cui non manca mai di appuntare lo sguardo" (Luzi, 1984, p. 47):

In qualche modo la verticalità del rapporto con la tradizione risarcisce la penuria di altri rapporti - o è proprio lei che li rende deboli e improbabili? A ben guardare la solitudine di Ungaretti ha un interlocutore continuo: è appunto la tradizione in tutte le variabili che il sentimento di essa può riaccendere, inclusi provvisoriamente certi orpelli. Credo che su questo punto sia il caso di insistere più di quanto, sulla scorta degli stessi scritti letterari del poeta, si sia fatto fin qui: non si tratta solo di un tema quantunque fondamentale alla messa a punto di un criterio morale ed estetico e neppure di un binario su cui corre la ricerca stilistica: si tratta, né più né meno, del movente primario. (...) 
L'essere nato nella diaspora, cresciuto in una povera comunità d'emigranti, in un paese senza colori precisi aperto a promiscuità e a disperazione, alibi l'una e l'altra, lì proprio ai margini del deserto che è anche un abisso del tempo non può non avere ingenerato nel giovane Ungaretti uno sperduto e avventuroso senso di apolidismo: un senso ambiguo a cui egli associa un sentimento controverso di libertà e di deiezione. (Luzi, 1984, p. 47)

L'esperienza del nomadismo, o in termini luziani dell'essere "apolide", scatena per reazione contraria la ricerca delle origini, "tutto quanto si riassume nel concetto totemico di tradizione, (...) la polarità opposta del luogo proprio, dell'atavismo, della continuità culturale, della patria dunque e della civiltà che ne è nata” (Luzi, 1984, p. 48), con un'intensità e con un approccio drammatico particolare e opposto a chi della tradizione invece vuole liberarsi:

Conoscere la propria tradizione, approfondire il senso, entrare a farne parte non ha per chi ha fatto sua la condizione del nomade lo stesso valore, ammettiamolo, che ha per chi dall'interno deve prenderne coscienza e, magari, liberarsi dal suo peso. In Ungaretti, come sappiamo tutti, non è impossibile trovare qualche accento di doloroso ulissismo prima che l'inserzione nella realtà della patria mediante battesimo di fuoco e d'acqua abbia avuto il suo tempo e il suo luogo negli anni di guerra, tra i fiumi e le doline del Carso e nel naufragio di una civiltà appena riconosciuta. (Luzi, 1984, p. 48)

Per rendere meglio l'idea di tale rapporto con il portato dei suoi predecessori, Luzi utilizza l'immagine della tradizione come una casa nella quale il poeta ambisce d'entrare per prendere consapevolezza di quanto è stato privato:

Se il fulcro dell'opera di Ungaretti è il rapporto e meglio sarebbe dire con parole più sue il sentimento della tradizione, che cos’è la tradizione secondo Ungaretti, oltre ad essere, come dicevamo, la casa dove il girovago ambisce di entrare se non per misurare il senso della sua privazione? Dopo lo spaesamento e l'umile sofferta estraneità e le elementari agnizioni del tempo dell' Allegria, al 
poeta che ha messo - è il caso di dirlo - sanguinose radici in Europa e in Italia la tradizione si presenta come un universo da riconoscere e ripensare dal suo interno. (Luzi, 1984, p. 48)

La tradizione è recuperata sul campo del lavoro, nel senso già indicato di scavo nella parola, di recupero del suo significato, conoscenza fattuale, mai in termini d'astrazione teorica o mera definizione, in una lettura assolutamente libera da condizionamenti o presupposti ideologici, fatta di elezioni e ripulse già eloquenti ed espressive in sé:

È vero che niente affatto portato all'astrazione concettuale Ungaretti si astiene dal dare e anche dal cercare la definizione come allora, in tempo di “rappe à l'orare". Tutto o quasi è demandato ai suoi orientamenti di fatto e dunque principalmente di lavoro. Lo studio insistente e ricorrente dei suoi autori elettivi Petrarca, Leopardi, Allarme, Gongola è la più scoperta tra le possibili indicazioni. E le ripulse, talora nette come nel caso del surrealismo, non sono meno eloquenti. Ma il tutto sarebbe assai poco e potrebbe anche dire il falso se non si avesse presente il modo di lettura di Ungaretti che è un modo assolutamente non vincolato né mediato da alcuna ideologia vigente, anzi bellamente in contrasto con tutte. (Luzi, 1984, p. 48)

A spingere Ungaretti verso la tradizione, prosegue Luzi, è "l'avidità di una conoscenza fattuale" (Luzi, 1984, p. 48), lo scavo "con ardente filologia d'artista" (Luzi, 1984, p. 48), l'investigazione "di elementi materiali profondi che fanno il valore, la memorabilità dell'opera" (Luzi, 1984, p. 48); Ungaretti aveva "un'idea operante della tradizione che si era fatto e che si andava continuamente facendo" (Luzi, 1984, p. 48), e che si manifestava nel "lavoro in concreto sui nuclei e sulla filigrana dei testi prediletti" (Luzi, 1984, p. 48), che impegnava "simultaneamente e reciprocamente l'invenzione e la riflessione, la poesia e il discorso su di essa, la tecnica e i movimenti” (Luzi, 1984, p. 48).

Il tema della tradizione per Ungaretti è "più vissuto che formulato" (Luzi, 1984, p. 48) e "non ha nulla a che vedere con il riconoscimento dell'autorità di un ordine obbligante" (Luzi, 1984, p. 48), mette al centro il valore della parola, "riconosce tutto il potere di rivelazione che ha la storia della parola umana e la storia laboriosa dei modi in cui essa ha superiormente parlato" (Luzi, 1984, p. 48). È questa un'idea geniale ed esplicita, ma 
per Ungaretti, sottolinea Luzi, "le idee contano assai meno che il rapporto vitale con esse" (Luzi, 1984, p. 48) e la sua poesia dimostra con estrema libertà la fattualità di tale idea, la sua concretezza:

Quando la libertà è al massimo del proprio regime riesce a generare il convincimento, abbastanza esaltante, che al di là del senso reale delle epoche la tradizione sia essa stessa una realtà più grande dei dati che la compongono; sia anzi un universo che l'uomo inventa per un atto di reciprocità tra passato e presente e si avvivi in uno spazio della memoria che viene più da lontano della storia. È, come dire?, l'invenzione del tempo dei tempi e cioè della loro attuale ricognizione in un punto (che è poi un'immensità) che li fa vivere tutti. (Luzi, 1984, p. 48)

Dopo aver assodato il legame Ungaretti / tradizione, Luzi si chiedeva quale fosse, "al di là del beneficio della sua grande maternità, il vero contributo di conoscenza e di luce che essa porta" (Luzi, 1984, p. 48) nella lirica di un poeta sempre diviso tra un "perpetuo non luogo" (Luzi, 1984, p. 48) e il suo riconoscersi "situato all'interno di una cultura" (Luzi, 1984, p. 48), e affermava:

Tra Ungaretti come poeta del perpetuo non luogo (la cui condizione di nomade era divenuta fatalmente simbolica di un'età senza certezze) a poeta che si riconosce situato all'interno di una cultura e diventa appunto poeta colto perché agisce in un continuo paragone con quella cultura, con quella tradizione, si apre una partita abbastanza difficile tanto più che né l'uno né l'altro aspetto è rigidamente chiuso nel libro che contrassegna questa o quella stagione della poesia ungarettiana. Né l'Ungaretti della ansiosa deriva è tutto nell' Allegria né l'Ungaretti reintegrato nel discorso europeo e italiano è tutto nel Sentimento o nel Dolore. (Luzi, 1984, p. 48)

La lirica d'Ungaretti si gioca, secondo Luzi, nel mai appagato e altalenante oscillare tra "spaesamento" (Luzi, 1984, p. 148) e "appartenenza profonda" (Luzi, 1984, p. 148), in un'inquietudine mai placata neppure dal recupero della tradizione: 
La scoperta della tradizione civile letteraria e religiosa e il lavoro della mai soddisfacente appropriazione del suo significato non acquietano l'ansietà di Ungaretti il quale per l'appunto sembra fondere i suoi temi dialettici fondamentali dello spaesamento e dell'appartenenza profonda, del moto necessario e della stasi desiderata in un punto che è fuori della tradizione, ma è dove la tradizione stessa confina con il mito primordiale. (Luzi, 1984, p. 48)

Ne è prova, sempre secondo Luzi, il permanere nella Terra promessa di alcuni accenti poetici dell' Allegria, ovviamente con stratificazioni d'anni e d'esperienza, tra ricordo e celebrazione di condizioni e sentimenti mai veramente sperimentati o raggiunti, appena intravisti per momentanee e fulminee apparizioni:

Nella Terra promessa ritroviamo, sovraccarichi di tutti i sensi che l'età e la lunga prova hanno lasciato, gli stessi accenti dell'Allegria. È anzi un unico accento di commemorazione: commemorazione di un passato non identificato, commemorazione di una felicità non avuta, che rapidi e favolosi accecamenti del desiderio hanno fatto balenare come possibili, commemorazione di un'integrità non provata, di un'innocenza non conosciuta. (Luzi, 1984, p. 48)

Accade, infatti, che in Ungaretti quella che potrebbe diventare poesia funebre, per una mancanza, per un'assenza, per l'esperienza della morte, si trasformi in "innografia”:

Ancora, ma con più freddo nelle sue giunture, la parola trasforma quell'epicedio in una effimera innografia dell'origine e del primario come nell'Allegria. E infatti su quel passato senza volto è spuntata l'attesa. Ciò che è al di là del presente come miraggio è una riscoperta dell'antico. Futuro è primordio. Terra promessa è infatti terra del ritorno. Questo è appunto il dominio. Adesso senza più le difficili frizioni con il presente, della memoria. (Luzi, 1984, p. 48)

È una memoria particolare quella di Ungaretti, "senza oggetto su cui svettano due sole luci possibili, quella del mito e quella del desiderio" (Luzi, 1984, p. 48), da cui deriva un dettato poetico per nulla affine a quello di un poeta tradizionale, intendendo con tale 
termine colui "che la tradizione l'ha assimilata (...) criticamente come retaggio". (Luzi, 1984, p. 48) Il rapporto con la tradizione si gioca, per Ungaretti così come per tutti, nello scontro, collisione o colluttazione, che può essere rifiutata o accettata o rinnegata:

Sono due modi di partecipare al tumulto che Ungaretti sente convivere in sé: mitezza remissiva e alte lamentazioni si alternano da un punto all'altro della sua opera: la furia e la follia delle passioni sono opposte e profonde pacificazioni trovate nell'assoluta desistenza della volontà e dell'io nella bivalente maternità della vita e della morte. (Luzi, 1984, p. 48)

Luzi, ci tiene a sottolinearlo, non sta scrivendo unicamente a proposito di Ungaretti autore e poeta, o semplicemente dell'uomo Ungaretti, quanto piuttosto dell'"uomo fissato nella maschera ungarettiana, solo, su quella scena totale, priva di quinte storiche e di angolature prospettiche, è l'uomo riconoscibile di una situazione altrettanto riconoscibile" (Luzi, 1984, p. 48), poiché è fermamente convito che dalle liriche del poeta apolide emerga un uomo, non solo privo di uno spazio geografico certo, ma anche di uno spazio temporale preciso, messo alla prova del crogiuolo di una conflagrazione universale:

Questo cittadino del tempo ormai secolare della crisi e dell'insicurezza, bruciato adesso alle fiamme di una conflagrazione micidiale, fruisce di una dignità intemporale, si reintegra per opera di Ungaretti in una specie di Eternità. Religiosamente Ungaretti conferisce nudità e grandezza all'immagine dell'uomo sbalzato fuori dal suo supposto predominio, cristianamente Ungaretti esaspera e nello stesso tempo medica con pietà le ferite del suo protagonismo: suo, vale a dire dell'uomo di cui Ungaretti si investe, salvando per altro sempre una sua provvidenziale puerizia. (Luzi, 1984, p. 48)

Ungaretti, infatti, riflettendo sul proprio destino e quello dell'uomo moderno, in termini per così dire leopardiani, compie un'azione simultanea e sincronica: da un lato "solleva alla grandezza della visuale tragica propria della tradizione la miseria dell'uomo disingannato" (Luzi, 1984, p. 48), dall'altro "sgretola la sua squallida immagine, l'annulla nel niente del senso e della natura, serbatoi e cisterne dove la tradizione ha riposto l'essenza 
della continuità" (Luzi, 1984, p. 48); in altre parole è proprio in forza dell'esperienza dell'annullamento del senso e della natura che riparte il riscatto, il rinnovamento, la ripartenza della poesia alla ricerca dell'identità dell'uomo. Per dirla ancora con parole di Luzi:

Ungaretti dibatte alla grande secondo la misura leopardiana e nella nuda speculazione del destino, ma non senza astuzia di altre millenarie saggezze, un problema moderno. Offre infine questo individuo combattuto e disfatto all'elaborazione successiva quando, essendo in causa non più l'onore dell'uomo ma la sua identità, la poesia riparte rischiosamente, umilmente, alla sua riscoperta. (Luzi, 1984, p. 47)

L'intervento luziano appena ripreso, fu un contributo cardine tra quelli della critica ungarettiana, tanto che il medesimo intervento, in forma leggermente ridotta, è stato ripubblicato a piena pagina nel 2000, a trent'anni dalla morte del poeta "naufrago", sulla rivista Poesia, accanto ai contributi di colleghi, poeti e studiosi, quali Andrea Zanzotto, Vittorio Sereni e Silvio Ramat, tra i quali scelgo di soffermarmi sul saggio di Andrea Cortellessa, poiché accenna alla questione dell'eredità ungarettiana nei poeti successivi, ossia alla traccia lasciata dalla sua lezione poetica. Titolando il suo contributo all'insegna dell'ossimoro, in Il teatro naturale, ne rimarcava l'insolito controsenso tra il grande successo delle sue liriche da un lato, con le 75.000 copie vendute del Meridiano Vita d'un uomo. Tutte le poesie, curato da Leone Piccioni, e le successive sedici edizioni, che lo incoronarono senza dubbio "ultimo grande poeta popolare della nostra tradizione" (Cortellessa, 2000, p. 3) e la mancanza d'emuli o eredi, dall'altro; sembra che la sua lezione specificamente poetica resti apparetemente lettera morta, a confronto di Saba e Montale che ebbero "una schiera di prolificissimi 'nipotini'” (Cortellessa, 2000, p. 3). Cortellessa ne cercava una possibile spiegazione nell'"apparente facilità del verso breve dell'Allegria e del Dolore d'Ungaretti" (Cortellessa, 2000, p. 3), che in realtà nasconde "un febbrile lavorìo costruttivo, l'implacabile mulinìo di versi della tradizione frantumati, rinsaldati, di nuovo disgregati in una sorta di infinito laboratorio ritmico" (Cortellessa, 2000, p. 3) e proseguiva:

Non c'è niente da fare: per i poeti d'oggi (che magari da adolescenti avranno cominciato, come tutti, proprio dalla suggestione di quelle parole acuminate, 
splendenti in un mare di pagine bianche, a spezzare le righe delle loro piccole confessioni) Ungaretti resta troppo ingenuo, e al tempo stesso troppo difficile. È un paradosso arduo da spiegare, e che peraltro il poeta ha scientificamente perseguito lungo tutta la sua traiettoria (secondo il cruciale binomio di innocenza e memoria: titolo di una prosa datata 1926 che inaugura l'interpretazione critica dei poeti prediletti da sempre, Leopardi e Mallarmè). Lo dirà, Ungaretti, in parole semplici (e dunque misteriose), in un'intervista raccolta da Aldo Borleghini nel '61: "la poesia è un lavoro complesso, non è un lavoro semplice, anche se è frutto di ispirazione, e non può essere che frutto di ispirazione, ma l'ispirazione è il punto di partenza, e il punto di arrivo è di renderla ancora pura ispirazione. Se ci arriva, si fa la poesia”. Un’immensa tradizione - destinata a bruciarsi al fuoco di un'intensità vitale senza freni. (Cortellessa, 2000, p. 3)

Ungaretti anticipa con il tema del naufragio rielaborazioni poetiche e teoretiche che saranno proprie dell'esistenzialismo, ma ciò che lo contraddistingue e che lo rende difficilmente imitabile è la trasposizione della condizione di naufrago sul piano del poiein poetico: è infatti una sorta di naufragio semantico quello di Ungaretti, che lotta per riappropriarsi della parola in un mare di infinite possibilità semantiche, per dirla con Zanzotto:

È il linguaggio ungarettiano, quel linguaggio all'orlo dell' afasia, balbettamento di parola comune e insieme scansione lapidaria e "pura", che invera il tema esistenziale proprio in questa luce. (...) L'incontro con la parola "trovata" nel silenzio, "scavata come un abisso" nel seno stesso della vita, dell'essere, gorgo rilevante, "nulla d'inesauribile segreto", non introduce tanto, o solo, alla giustificazione di uno stile, quanto ad un diverso rapporto tra essere e dire. ̇̀ questa la parola della pietra, di quella pietra che l'uomo, che è l'essere: il peso, le implicazioni semantiche dei termini, il mondo del loro annunciarsi, per cui ogni frammento sembra strappato con immane fatica al silenzio definitivo, alla morte, introducono al tono di un'epoca dalle più cupe prospettive, in cui ancora oggi ci si trova. (Zanzotto, 2000, p. 4) 
Non si può dar conto in questa sede di tutti gli interventi critici relativi a Ungaretti, mentre può essere pertinente e interessante approfondire intersezioni tematiche, stilistiche, contenutistiche tra i due autori, andando ad indagare presenze o influssi ungarettiani, nella lirica luziana, a partire dal recente saggio critico di Enrico Elli, nel quale lo studioso evidenzia il ricorrere "di temi fondamentali per entrambi i poeti: il viaggio, la luce, la memoria dei defunti" (Elli, 2014, p. 138). Sul primo tema Elli articola una serie di esemplificazioni e parallelismi tra l'autore di Viaggio terrestre e celeste di Simone Martini e Vita d'un uomo a partire dal motivo della vita come viaggio, riferimenti testuali e reminiscenze ungarettiane comprese.

A partire dal titolo quello di Luzi e del suo alter-ego artistico, Simone Martini, si dichiara come un viaggio: storico, personale e metaforico. Storico nel senso manzoniano e bacchelliano del termine, perché nella breve didascalia iniziale Luzi stesso avverte di aver voluto integrare i "silenzi della storia" immaginando l'estremo viaggio del pittore da Avignone a Siena sua terra d'origine. Superfluo e addirittura apparentemente banale ricordare come l'intera raccolta poetica di Ungaretti, sotto il titolo complessivo Vita d'un uomo, sia costruita attorno al motivo della vita come viaggio. Ungaretti è poeta viator che proclama fin dagli esordi del Porto sepolto nella lirica programmatica Lindoro di deserto: "Sino alla morte in balia del viaggio"; e se anche incappa in naufragi "subito riprende / il viaggio / come / dopo il naufragio / un superstite / lupo di mare" (...). In prima istanza il viaggio di Simone-Luzi si configura, per la sua parte terrestre, come un nostos che intende congiungere la fine con l'inizio. Tuttavia, Luzi stesso confida: "Ed il viaggio è il nostos, ma non al punto di partenza, e Siena non è più Siena, è un'altra cosa” (Elli, 2014, p. 139).

Proseguendo la ricognizione, Elli evidenzia casi di memorabilità ungarettiana nel testo luziano a proposito dell'ambientazione paesaggistica e solare di Non girasoli, frumento di Luzi, segnalando rispondenze testuali con Di luglio da Sentimento del tempo, che qui si riporta:

Le immagini poi di Non girasoli, frumento ci presentano un paesaggio estivo non certo idilliaco, ma raccontano un'estate che brucia, spoglia e divora, e tutto rende simile a cenere: "Frumento raso / (...) / Brucia, estate, il suolo / spoglia giro giro / di alberi, di ombre / (...) / cenere (...) / Brucia, essa, l'aria / (...) / divora l'azzurro". Inoltre, tre volte Luzi parla della mattina di luglio e della sua luce. Non è chi non ricordi l'immagine ungarettiana dell'estate nel 
Sentimento del Tempo che della lirica Di luglio "Strugge forre, beve fiumi, I Macina scogli" e "Va della terra spogliando lo scheletro"; o la poesia D'agosto che presenta "prostrate messi" e "ceneri". Anche in questo caso, dunque, consonanza di immagini e soprattutto di sensazioni. La terra d'Orcia, a sud di Siena, diviene nuova tappa, ulteriore, forse definitiva pausa: "la sua oasi". (Elli, 2014, p. 138)

Lo studioso prosegue e approfondisce ulteriormente il significato del termine viaggio nei due autori in esame, precisandone la derivazione da Dante piuttosto che da Ulisse:

Per entrambi, tuttavia, il viaggio è ben più che un semplice ritorno. Esso diviene pellegrinaggio alle fonti della vita e dell'arte, a segnare una sorta di tempo circolare o, per meglio dire, l'assunzione del tempo umano nell'eterno presente dell'Essere. A proposito dell'ultima sezione Ispezione celeste, a Verdino che chiede nelle preziose conversazioni con l'autore nelle pagine A Bellariva. Colloqui con Mario: "Qui è la fine del viaggio, nel terrestre trasmutato in celeste?", il poeta risponde: "Qui è un dopo. La storia di Simone non finisce con Simone". A questo punto, non solo Siena ma l'intero Viaggio è diventato un'altra cosa; poiché se la terra orciana segna l'andare oltre nella linea dello spazio, la storia di Simone che non finisce con lui segna una direzione che va oltre nella linea del tempo. Questo superamento delle coordinate spazio-temporali ci proietta in una prospettiva che a me pare assimilabile all'ungarettiana Terra Promessa, a proposito della quale il poeta, al termine del suo percorso esistenziale e poetico, negli Ultimi cori per la Terra Promessa conferma: "Non d'Itaca si sogna / Smarriti in vario mare, / Ma va la mira al Sinai". Non, quindi, un ritorno ulissidico, ma un viaggio che presenta altre coordinate, prime fra tutte quelle dantesche. (Elli, 2014, p. 139)

Sul tema delle rispondenze tematiche tra i due autori e l'idea dantesca di viaggio si rimanda al saggio di Elli e, per non uscir di traccia, si procede in autonomia ad evidenziare qualche altro spunto d'affinità tra le liriche dei due poeti. È il caso, ad esempio, di 
considerare la consonanza nell'uso della versificazione tra il primo Ungaretti e l'ultimo Luzi. È noto a tutti quanto sia stato importante l'uso dello spazio, la riduzione al minimo della punteggiatura, dell'alternanza di spazi bianchi e scritti, di pieni e vuoti nella lirica di Ungaretti; si è messo in evidenza quanto lo spazio bianco tipografico a separazione tra due strofe lo fosse altrettanto per Luzi fin dai suoi esordi, con il riferimento nel capitolo precedente alla precisazione pubblicata su Frontespizio all'uscita di alcune sue liriche inedite recensite da Fallacara ed erroneamente disposte sulla pagina senza partizione in strofe annotava:

Una poesia concepita per strofi intende di essere più vasta di quanto il numero secco dei versi le permetterebbe. In ogni strofe, specialmente nella quartina quale io immagino, l'emotività e il suono dovrebbero coincidere in un acme da cui è impossibile discendere al silenzio in un giro di sillabe così breve. Ed allora avviene che le quantità emotiva e sonora eccedano su quella verbale. Dev'essere l'intervallo, lo spazio bianco, a permettere una completa distensione musicale; talvolta una moltiplicazione indefinita di vibrazioni. (A questo giovano alcune parole scelte tra quelle insufficienti a definire un suono o una suggestione, idonei invece a rifletterli e amplificarli, parole alveari, da porsi alla fine della quartina). Ma non ho alcun diritto di rivelare il mio silenzioso mestiere, le mie esperienze stilistiche non possono che avere il volto attribuito loro dagli altri. Dicevo per quello spazio bianco che mi è stato sottratto. (Luzi, 1937, p. 471)

La studiosa Elisa Tonani se ne è occupata ad ampio raggio in due capitoli distinti, dedicati ai due autori, nel suo studio Punteggiatura d'autore (2012) Il ritmo ascendente di un discorso, così come nel saggio intitolato Il ritmo ascendente di un discorso frammentario, nei quali delineato il verificarsi nella metrica luziana, a partire da Nel magma in forma germinale e poi via via con modalità eclatante, l'introdursi nella lirica luziana di una "espansione, di una dilatazione di strutture formali dirompenti rispetto a quelle chiuse di una tradizione lirica da cui l'opera di Luzi ha preso le mosse" (Tonani, 2014, p. 93), visibili in modo chiaro proprio nello specifico della disposizione tipografica del testo poetico.

È inevitabile notare che i due autori a confronto abbiano svolto un cammino inverso, un viaggio in direzione opposta rispetto alla tradizione: Luzi vi era immerso, toscano nella sua Toscana, tra Firenze e Siena, tra Dante e Petrarca, ed era impegnato in un 
diuturno scavo, uso, approfondimento, studio e rielaborazione della stessa, dei suoi testi, delle sue forme e dei diversi stili in un continuo lavoro di riflessione critica sui poeti e la letteratura, indotto anche dalla professione d'insegnante: il suo è un viaggio letterario che a partire da una piena conoscenza e consapevolezza del portato della tradizione la innovava e se ne allontanava gradualmente, osando sperimentare e contaminare, a partire anche da suggestioni altre.

All'opposto, come già indicato in precedenza, Ungaretti si riappropria di una tradizione mancante, compiendo un viaggio reale e di carta, in un percorso di stile e d'uso metrico dal semplice e frammentario al complesso, per approdare alla versificazione della tradizione. Un percorso inverso compiuto in nome della parola, dello scavo e della ricerca del suo massimo significato, a partire dal frammento come incipit ed explicit del proprio versificare, poiché, per dirla con Elisa Tonani, "il frammento non è soltanto una diminuzione della continuità, ma è anche una risorsa che testimonia la fiducia nella parola, la quale, isolata nel bianco, tende all'assoluto, alla perfetta coincidenza con il Verbo" (Tonani, 2012, p. 93).

Richiamandosi ad alcuni scritti luziani su Mallarmè, la studiosa ribadiva il valore demiurgico dello spazio bianco, la sua forza di creazione, intesa con valenza di discorso continuo, di voce che interrompe il silenzio, di frammento che frange la pagina bianca:

Il bianco che alona esigui frammenti di verso in qualche modo ostenta un residuo di demiurgia analogo a quello che Luzi attribuisce a Mallarmé quando scrive che "il poeta che si era messo alla caccia del Verbo o poteva limitare il valore attivo della parola tanto da farlo coincidere con la nozione grammaticale di verbo. Verbi sono anche altri termini del discorso quando il poeta gliene attribuisce il valore e il potere". Il bianco serve proprio ad attribuire valore e potere linguistico-semantico a termini che normalmente fungono da connettivi, da determinanti, da modificatori di altri termini, e che non hanno una forza autonoma. Il bianco gioca infatti sulla possibilità di mostrare la parola prima della sua aggregazione nel discorso, per così dire nel suo sorgere, nel suo isolamento di epifania, contornata solo dalla luce che la dis-vela. Ma c'è anche, accanto a questo aspetto, un uso delle spezzature tipografiche da 
intendersi come mimesi del movimento, della faticosa progressione del senso (ancor più che come ricerca di effetti di assolutezza). (Tonani, 2014, p. 97)

Seguendo la traccia del discorso della Tonani si scopre un'altra annotazione importante e preziosa ai fini del presente confronto, che chiama in causa anche Caproni:

Se i bianchi di Ungaretti sono silenzi intensificatori della parola, e quindi coincidono con il massimo del potenziamento, e i bianchi di Caproni comportano una riduzione della struttura testuale, un indebolimento dell'organizzazione logico-sintattica, Luzi imbocca una terza via in cui il bianco è tra l'altro un modo per segnalare che il discorso ha una progressione ansimante, intermittente, ovvero procede per tappe: come se ogni volta si prendesse il respiro per compiere il passo successivo (e davvero per la sua opera poetica funziona molto bene il figurante metaforico del viaggio). (Tonani, 2014, p. 97)

Da tale raffronto si coglie che l'uso isolato della parola, potenziato tra spazi bianchi, svolto dal primo Ungaretti in una sorta di recupero e scavo e ricerca della "lirica pura", come ampiamente indagato da un nutrito numero di critici e studiosi, in primis da quelli d'area ermetica, e soprattutto da Carlo Bo, del quale si riporta uno stralcio tratto da Dimora della poesia:

Rifacendosi da una povertà che non era una maniera ma la conclusione d'un atto di coscienza senza polemica, separato dalla violenza dell'aria, partendo dalla pagina bianca non già come ultima soluzione d'un'insuperabile possibilità di vita assoluta il primo lavoro d'Ungaretti ha dovuto essere quello della parola, e si badi non d'una parola essenziale come finora s'è detto ma d'una parola comune pura dei vizi del tempo, schiava dei desideri del poeta, della parola non più limite artificiale ma proprio come massima probabilità d'allusione e scansione naturale d'un nostro presente poetico insospettato. Nelle sue rare e decise confessioni di autore, Ungaretti ha preferito insistere su questo lavoro come su una necessità di mestiere: siamo d'accordo, ma vale la 
pena di ricordare qui che mestiere rimane per lui nell'intero possesso della sua dignità: di "mestiere d'uomo", di coscienza che va oltre il calcolo della fortuna pratica. (Bo, 1938, p. 149)

Così come da Leoni Piccioni, che ne aveva curato la prima edizione nei "Meridiani" Mondadori, e da De Robertis che si era occupato in tal senso delle varianti nella sua lirica, riemerge in modo affine un percorso al contrario nell'ultimo Luzi.

Se Piccioni coglieva i mutamenti nella versificazione ungarettiana nel passaggio dalla dissoluzione alla ricostruzione delle forme della metrica tradizionale ${ }^{5}$, Pier Vincenzo Mengaldo si era soffermato invece sulla portata innovativa del primo suo poetare, collegandola all'extraterritorialità culturale del poeta d'Alessandria d'Egitto, sulla cui metrica rivoluzionaria annotava:

Strumento fondamentale di questa rivoluzione è la metrica dell'Allegria: che disgrega il verso tradizionale in versicoli, frantumando il discorso in una serie di monadi verbali sillabate quasi come attonite intersezioni liriche (con Contini: "in Ungaretti il discorso nasce successivamente alla parola”). Ne viene dilatata la forza evocativa e impressiva del singolo vocabolo - che può essere quindi attinto di norma a un lessico del tutto normale, anti-letterario-, semantizzando anche parole vuote ( $d i$, una, come, e possono addirittura costruire da sole un verso) e coinvolgendo nella significazione ben più profondamente che nella poesia tradizionale pause di silenzio e spazi bianchi, marcati e resi ancora più polivalenti dall'apollinariana assenza di punteggiatura. (Mengaldo, 1978, p. 383)

Ecco che Luzi attua, invece, un percorso inverso innovando la tradizione con guizzi momentanei di novità metrica nella piena maturità, o meglio nella vecchiaia incipiente, guizzi che permettono d'individuare in verticale, in una sorta di doppia versificazione, la presenza

5 Leone Piccioni nella sopracitata Prefazione scriveva: "Certo la metrica, distrutta nei versicoli (anche se per ejambement, già mirabilmente risorgeva, in quinari, novenari, endecasillabi, come il De Robertis splendidamente mostrò, nel saggio del ' 43 sulla formazione della sua poesia) pareva proporre un tipo di discorso poetico, quasi del tutto nuovo, a dimensione diretta, colloquiale, popolare: ed era, invece, in quella drammatica situazione umana, in quella guerra dei fanti, misura eroica” (2009, p. XXIII). 
di un duplice testo poetico simultaneo nel medesimo componimento poetico; si riscontra, infatti, accanto alla "versificazione a gradino" documentata dalla Tonani, il coesistere nel medesimo componimento della tradizione accanto all'innovazione: la versificazione ora scorre nel solco di una metrica canonizzata di settenari, novenari e decasillabi, sul lato sinistro della pagina, ora invece si frantuma in quinari o in brevissimi versi bisillabi sul lato destro della stessa, in una straordinaria affinità con quanto Mengaldo aveva annotato a proposito della "metrica franta dell' Allegria" (Mengaldo, 1978, p. 383), ossia "l'equivalente prosodico di quella ricerca della parola 'nuda' ed essenziale (...) in cui sta la maggior novità, umana prima che stilistica, della raccolta” (Mengaldo, 1978, p. 384).

Se ne fornisce un esempio con la lirica seguente, tratta significativamente da Dottrina dell'estremo principiante:

Ha la sua giusta canicola

qui la solitudine. Deflagra

la mente

sovrana che ci pensa.

Lei soltanto.

L'altro non c’è.

L'altro

non è altro

da niente altro, è sé.

Si empie

di tempo la giornata,

si estingue nella sua durata

e così il suo ritorno.

Romba

qualche raro

motore sotto sforzo

su queste aride poggiate,

altrimenti tace il giorno.

Quella versificazione franta che si distingue e si isola verso il lato destro della pagina, che spicca grazie al bianco dell'impaginazione, permette al lettore d'individuare un 
altro percorso di lettura rapido, per balzi semantici, essenziale in verticale, che ha una sua unità logica e stilistica come qui si restituisce nella sequenza: Lei soltanto / L'altro / Si empie / Romba.

Similmente accade in altre liriche tratte dalla Dottrina, come si nota nella lirica seguente, dove la disposizione grafica permette nuovamente una concatenazione di termini in verticale sul lato destro del componimento in una progressione dal buio alla luce che riassume il senso profondo della lirica: Nero / ne bruciano / L'alba / ne arde / È un'alba / l'uomo / a che richiamo? / Veniat:

Notte. Nero

il recesso da cui s'alza

lei in aperto cielo -

$$
\text { ne bruciano }
$$

la brullicante azzurrità

le stelle,

i loro firmamenti.

$$
\text { L'alba }
$$

è lontana. Preme

però, oscura vampa,

nero-viola,

un lumine segreto, ne arde

tutto il campo

celeste inseminato,

e arde la sua ombra.

$$
\text { È un'alba }
$$

notturna quel fervore,

l'anima del mondo,

gli angeli, i deva, l'uomo

ed il suo grumo

rispondono, Porfirio, a che richiamo?

o fonde, si sbriciola in se stessa

la creazione, per un'altra 
più prossima al creatore...

Veniat.

Similmente si registra un percorso affine nella lirica che di seguito si riporta dove il poeta descrive un cammino dallo sradicamento attuato dalla "ventata" della vita al ritorno nel suo "grembo":

Toccò a lei, ultima ventata,

sradicarmi da dov'ero

lì a dimora.

Il tempo successivo

arò il mio corpo dalla mia memoria,

e spense ogni sentore,

sarchiò quel territorio

e fu materia

nella materia

il mio sfatto sudario,

si dissolse il grumo

casuale o necessario -

non so ancora -

che era stato

il mio nido,

il mio calvario,

se non che celeste e tenebroso

era nel suo deliquio

l'esistente provvisorio.

Oh vita, ascoltai quella loquela

provenire da te,

dal tuo profondo grembo. 
Si attesta struttura simile a doppia colonna, in una sorta di doppia voce dialogante, anche nella lirica successiva alla precedente, nella quale si registra una concatenazione di annotazioni tra la luce e il buio fino ad una domanda dubitosa di resurrezione:

Dal buio lungofiume

$$
\begin{aligned}
& \text { e dai suoi rari } \\
& \text { fari e lampade }
\end{aligned}
$$

si allunga, lo raggiunge,

lo supera da tergo

$$
\text { un'ombra, }
$$

$$
\text { di chi è }
$$

quell'ombra? si confonde con la sua

fino a che la ingoia

tutta quanta e n'è

totalmente fagocitata.

Cielo, a chi appartiene quella massa oscura

che obliqua si defila

da sotto quel chiarore

accecante di lampione?

a sé o all'altro che lo incalza

e sempre più da presso lo tallona,

lo investe, lo raggira?

o no, a nessuno,

$$
\text { individualmente, }
$$

ombra

o coacervo corporale

di tante differenze

e solitudini umane

che procede

verso l'annullamento,

lo scempio,

la resurrezione regalata? 
L'esemplificazione potrebbe proseguire oltre e superare il confine della raccolta Dottrina dell'estremo principiante, con sconfinamenti e riaffioramenti in altre raccolte, ma in questa se ne attesta una ricorrenza frequente suppongo non casuale e in linea con la pratica di un estremo principiante, che concreta nel verso e nella sua mise en page la vicissitudine della vita nella forma.

Nuovamente principiante, sebbene quasi ottuagenario, Luzi sovverte la tradizione o meglio la trasforma, mettendo in atto sulla soglia della fine del suo percorso poetico quella ricerca che aveva fatto del primo Ungaretti un innovatore, e facendo coesistere nel medesimo componimento poetico una versificazione compatta accostata ad un'altra tipologia metrica che sembra distruggere il verso per riscoprire il valore autonomo della parola, così come attuò Ungaretti ai suoi esordi, secondo la lettura che ne fece Giuseppe De Robertis in un saggio dedicato allo studio non solo delle varianti lessicali ma anche di quelle metriche e ritmiche:

Si tratterebbe dunque, più che di futurismo, d'una esasperazione di quella sua volontà di riduzione e concentrazione che già ai primi anni fruttò a Ungaretti acquisti originali. Solo che, perché questi non restassero un dono gratuito, un portato del semplice istinto, egli dié allora principio alla sua fatica vera, lavorando, si può dire lui solo, per tutta una generazione. Distrusse il verso per poi ricomporlo, e cercò i ritmi per poi costruire i metri. Tutta la musica della poesia ungarettiana, nelle sue infinite modulazioni, si sprigiona da questo suo farsi graduale, da quest'ascoltazione sempre più all'unisono col proprio animo, di cui le varianti e rielaborazioni sono la storia illustre. Nel distruggere il verso, nel cercare i nuovi ritmi, prima di tutto mirò alla ricerca dell'essenzialità della parola da ogni incrostazione sia letteraria che fisica. (De Robertis, 2009, p. 410)

Quel che Ungaretti attuò nell'uso di varianti e rielaborazioni, "prova di un'acuta, inquieta e, alla fine, vittoriosa ricerca dell'espressione, in una infinita scala di gradazioni" (De Robertis, 2009, p. 410), tangibile prova di un "graduale alleggerimento" (De Robertis, 2009, p. 410) del verso, per dirla ancora con De Robertis, si attua anche nella lirica di Luzi, nella sua versificazione un lavorio e una sperimentazione metrica che accentua e 
amplifica l'espressione semantica; a partire, infatti, dalla raccolta Per il battesimo dei nostri frammenti inizia a sfrangiarsi il verso ora nella struttura della versificazione a gradino, ora nella disposizione isolata di singoli vocaboli o brevi versi a destra della pagina con una tecnica di isolamento ed illuminazione della parola in linea con l'epigrafe tratta dal Prologo del Vangelo di Giovanni e riportata in calce alla raccolta: "In lei [la parola] era la vita; e la / vita era la luce degli uomini” (Luzi, 1988, p. 503). 


\section{Referencias}

Bo, C. (1938). Dimora della poesia. In Otto studi (pp. 149-230). Firenze, Italia: Vallecchi. Bo, C. (novembre, 1953). Un grido e paesaggi. Fiera Letteraria, VII(44), 5-6.

Bigongiari, P. (novembre, 1953). La terra promessa: inoltrandosi nel suo "interminato" poetico. Fiera Letteraria, VII(44), 3-4.

Cecchi, E. (novembre, 1953). Il dolore: "lo slancio muto della tua speranza". Fiera Letteraria, $\operatorname{VII}(44), 3$.

Cortellessa, A. (luglio/agosto, 2000). Giuseppe Ungaretti. Il teatro naturale. Poesia. Mensile Internazionale di cultura poetica, XIII(141), 3.

De Robertis, G. (novembre, 1953). L’Allegria: girovago su questa terra. Fiera Letteraria, $\operatorname{VII}(44), 3-6$.

De Robertis, G. (2009). Sulla formazione della poesia di Ungaretti. In C. Ossola (Ed.), Vita d'un uomo. Tutte le poesie (pp. 404-421). Milano, Italia: Mondadori.

Elli, E. (2014). Presenze ungarettiane nel Viaggio terrestre e celeste di Simone Martini. En P. Baioni \& D. Savio (Eds.), Un viaggio terrestre e celeste. Con un'appendice di scritti dispersi (pp. 137-146). Roma, Italia: Edizioni di Storia e Letteratura.

Gargiuolo, A. (novembre, 1953). Sentimento del tempo, la "situazione" di un artista. Fiera Letteraria, $\operatorname{VII}(44), 3$.

Luzi, M. (giugno, 1937). Emendamenti. Il Frontespizio, XV(5), 471.

Luzi, M. (1952). Profilo di Ungaretti. In V. Volpini (Ed.), Antologia della poesia religiosa italiana contemporanea (pp. 134-136). Firenze, Italia: Vallecchi.

Luzi, M. (novembre, 1953). Religiosità come evasione. Fiera Letteraria, VII(44), 4.

Luzi, M. (1979). Classicità e giubilazione di Ungaretti. In G. Grana (Ed.), Novecento. I contemporanei. Gli scrittori e la cultura letteraria nella società italiana (Vol. IX, pp. 8076-8079). Milano, Italia: Marzorati.

Luzi, M. (1984). La presenza l'attualità di Ungaretti. In Discorso naturale (pp. 41-49), Milano, Italia: Garzanti.

Luzi, M. (1988). Tutte le poesie. Milano, Italia: Garzanti.

Luzi, M. (luglio/agosto, 2000). Ungaretti e la tradizione. Poesia. Mensile Internazionale di cultura poetica, XIII(141), 8-10.

Luzi, M. (2002). Ungaretti e il suo infinito. In D. Piccini \& D. Rondoni (Eds.), Vero e verso. Scritti sui poeti e la letteratura (pp. 122-124). Milano, Italia: Garzanti. 
Luzi, M. (2002). Classicità e giubilazione di Ungaretti. In D. Piccini \& D. Rondoni (Eds.), Vero e verso. Scritti sui poeti e la letteratura (pp. 125-128). Milano, Italia: Garzanti.

Macrì, O. (1941). Esemplari del sentimento poetico contemporaneo. Firenze, Italia: Vallecchi. Macrì, O. (1968). Realtà del simbolo. Poeti e critici del Novecento italiano. Firenze, Italia: Vallecchi.

Mengaldo, P. V. (1978). La metrica rivoluzionaria di Ungaretti. In Autore, Poeti italiani del Novecento (pp. 381-384). Milano, Italia: Mondadori.

Ministero dei Beni Culturali a Roma. (1997). Ungaretti. La biblioteca di un nomade. Versione digitale della mostra allestita a Roma, Teatro dei Dioscuri 10 dicembre 199710 febbraio 1998. In Internet Culturale. Disponibile da http://www.internetculturale. it/opencms/opencms/it/pagine/mostre/pagina_425.html

Papini, G. (novembre, 1953). Un soldato semplice ha scritto le sue poesie: il primo articolo scritto su di lui. Fiera Letteraria, VII(44), 4.

Parronchi, A. (novembre, 1953). Interprete di poeti: il prosatore. Fiera Letteraria, VII(44), 5.

Pea, E. (novembre, 1953). Ungaretti in Egitto: il più giovane ospite della baracca rossa. Fiera Letteraria, $\operatorname{VII}(44)$, p. 4.

Piccioni, L. (2009). Prefazione. In C. Ossola (Ed.), Vita d'un uomo. Tutte le poesie (pp. XIIILV). Milano, Italia: Mondadori.

Raimondi, G. (novembre, 1953). Una scrittura tutta immagini: il prosatore. Fiera Letteraria, $\operatorname{VII}(44), 5$.

Ramat, S. (luglio/agosto, 2000). Il naufrago e il nomade. Poesia. Mensile Internazionale di cultura poetica, XIII(141), 10.

Sereni, V. (luglio/agosto, 2000). In morte di Ungaretti. Poesia. Mensile Internazionale di cultura poetica, XIII(141), 4.

Tonani, E. (2012). Punteggiatura d'autore. Interpunzione e strategie tipografiche nella letteratura italian a del Novecento ad oggi. Firenze, Italia: Cesati.

Tonani, E. (2014). Il ritmo ascendente di un discorso frammentario. En P. Baioni \& D. Savio (Eds.), Un viaggio terrestre e celeste. Con un'appendice di scritti dispersi (pp. 91105). Roma, Italia: Edizioni di Storia e Letteratura.

Ungaretti, G. \& Amrouche, J. (1972). Chi improvvisato. Parigi, Francia: Gallimard.

Ungaretti, G. (2009). Vita d'un uomo. Tutte le poesie (Ed. C. Ossola). Milano, Italia: Mondadori. 
“IL SENTIMENTO DELLA TRADIZIONE”: LUZI LEGGE UNGARETTI

Zanzotto, A. (luglio/agosto, 2000). Ungaretti: Terra Promessa. Poesia. Mensile Internazionale di cultura poetica, XIII(141), 4-5. 